\title{
Shear flow, viscous heating, and entropy balance from dynamical systems
}

\author{
TAmás TÉl $\left({ }^{*}\right)^{1}$, JÜrgen Vollmer $\left({ }^{* *}\right)^{2,3}$, and LÁszló MátyÁs $(* * *)^{1}$ \\ 1 Inst. Theor. Physics, Eötvös University, P.O. Box 32, H-1518 Budapest, Hungary. \\ 2 Fachbereich Physik, Univ.-GH Essen, 45117 Essen, Germany. \\ 3 Max Planck Institute for Polymer Research, 55128 Mainz, Germany.
}

PACS. 05.70.Ln - Non-equilibrium thermodynamics, irreversible processes.

PACS. 05.45.Ac - Low-dimensional chaos.

PACS. 51.20.+d - Viscosity, diffusion, and thermal conductivity.

\begin{abstract}
A consistent description of a shear flow, the accompanied viscous heating, and the associated entropy balance is given in the framework of a deterministic dynamical system, where a multibaker dynamics drives two fields: the velocity and the temperature distributions. In an appropriate macroscopic limit their transport equations go over into the Navier-Stokes and the heat conduction equation of viscous flows. The inclusion of an artificial heat sink can stabilize steady states with constant temperatures. It mimics a thermostating algorithm used in non-equilibrium molecular-dynamics simulations.
\end{abstract}

Introduction. - In the last years, there has been an increasing interest in modeling transport phenomena by low-dimensional, deterministic dynamical systems [1] 13]. Multibaker maps $[1$ 13] appeared to be the simplest models of this approach. They provide an opportunity to derive the equations of non-equilibrium thermodynamics from an underlying dynamics without using the concept of particles. The strongly-chaotic mixing properties of these twodimensional maps seem to be sufficient to ensure consistency with the entropy balance equation of thermodynamics provided that a properly chosen coarse-grained entropy and a macroscopic limit are taken [7, 10, 11]. Previous work successfully described the phenomena of diffusion [4, [6], conduction in an external field [5, 4, 9], chemical reactions [8], thermal conduction [10], and cross effects due to the simultaneous presence of an external field and heat conduction 11] by means of multibaker maps. Not only stationary, but also transient states could be addressed [7, 11, 12]. Here we add to this list the phenomenon of shear flows and the accompanying viscous heating. The interest of this is to clarify how the shear rate enters the expression for the irreversible entropy production. After all, by the definition of local equilibrium the macroscopic flow profile does not appear in the entropy balance. Thermodynamic averages contain only deviations from the average streaming velocity.

$\left({ }^{*}\right)$ E-mail: tel@poe.elte.hu

(**) E-mail: vollmer@mpip-mainz.mpg.de

$(* * *)$ E-mail: matyilac@poe.elte.hu

(c) EDP Sciences 
(a)

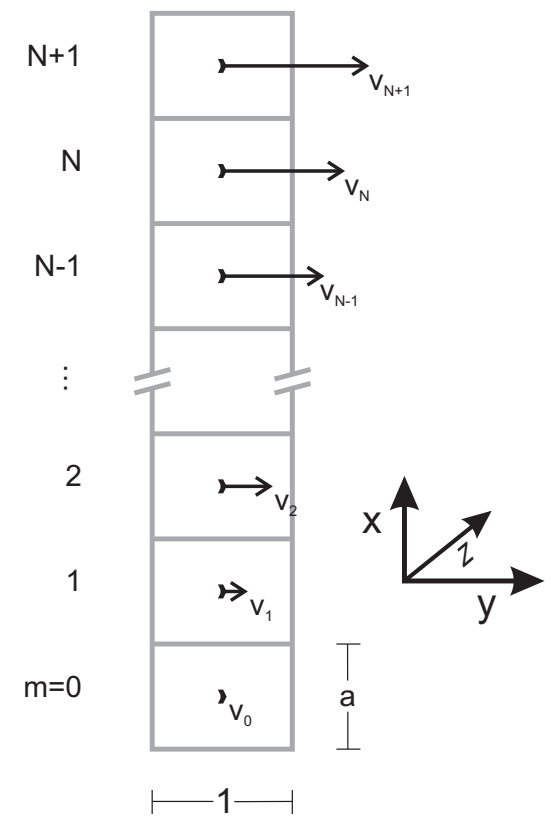

(b)

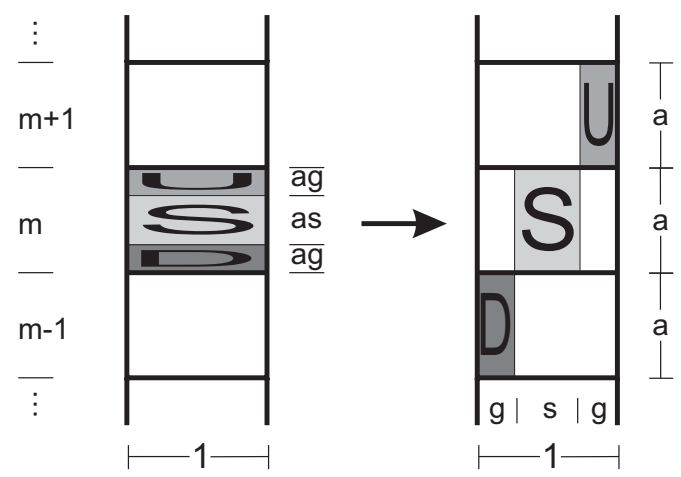

Fig. 1 - The multibaker map. a) The chain of $N+2$ baker cells of height $a$ and of unit width modeling an intersection of the fluid along the $x$ axis. The arrows indicate the average velocity $v_{m}$ of the fluid at positions $x \in[a m, a(m+1)]$. b) The 'microscopic' dynamics of cell $m(0<m<N+1)$. All notations are explained in the text.

We consider a viscous fluid driven by applying a shear forcing to two parallel walls of separation $L(c f$. 14, 15]). The fluid is assumed to be incompressible. The upper wall moves in the $y$-direction with a constant velocity $U$ (fig. 1 1a). We restrict ourselves to a discussion of (possibly non-stationary) laminar flow. Due to translation invariance the velocity $v$ is always directed in the $y$ direction, and it only depends on the $x$ coordinate. Similarly, the temperature $T$ is independent of the $y$ and $z$ coordinates. There is no external pressure gradient applied, and the internal pressure forces due to eventual temperature changes are expected to be negligible. For sufficiently long times the well-known linear velocity profile $v^{*}(x)=U x / L$ is approached as asymptotic steady state. The spatial behavior of the temperature distribution $T(x, t)$ depends on the boundary conditions. Initially $T$ is typically increasing in time due to the viscous heating. For an adiabatically closed fluid, the asymptotic distribution $T^{*}$ is spatially constant and grows linearly with time. Inclusion of a heat sink can remove this time dependence such that the asymptotic state becomes stationary. In a hydrodynamic setting the sink acts only at the boundaries of the system, but an artificial, spatially-uniform sink can also be implemented. It mimics the so-called SLLOD [16] algorithm of non-equilibrium molecular dynamics.

A multibaker map for shear flow. - We model an intersection of the fluid along the $x$ axis by a multibaker map. It consists of $N+2$ cells of size unity times $a$ (fig. 1 1a). The cells $m=0$ and $N+1$ represent the boundary, and cells $m=1, \ldots, N$, the bulk of the fluid, whose width is $N a=L$. In each cell there is a momentum-like variable $p$ defined besides the position. The multibaker dynamics advects two fields; the 'microscopic' velocity $v(x, p ; t)$ and the 'microscopic' temperature $T(x, p ; t)$. The appropriate ( $c f$. below) averages $v_{m}$ and $T_{m}$ 
over cell $m$ are called the coarse-grained fields. In the macroscopic limit they go over into the hydrodynamic velocity field and the thermodynamic temperature, respectively.

The two-dimensional multibaker dynamics is defined as follows (fig. 1 $\mathrm{b}$ ). After each time unit $\tau$ every cell $m=1, \cdots N$ is divided into three bands of heights $a g$, as and $a g$, such that $g+s+g=1$. The outermost ones are mapped onto a column of height $a$ and width $g$ in cells $m+1$ and $m-1$, respectively. The middle one stays in cell $m$, where it is transformed into a column of width $s$. In all cases the area is preserved such that an initially constant phase-space density $\varrho$ remains constant in time, reflecting the incompressibility of the fluid. Hence, the density can be identified with the density of the fluid.

The velocity and the temperature fields are advected by the multibaker dynamics. In contrast to the density $\varrho$ they typically evolve in time. Starting with a coarse-grained velocity distribution $v_{m}$ along the chain, the updated values $v_{m}^{\prime}$ after time $\tau$ become

$$
v_{m}^{\prime}=v_{m}+g\left(v_{m-1}+v_{m+1}-2 v_{m}\right)
$$

This update expresses momentum conservation in the $x$-direction. A portion $s=1-2 g$ of the original momentum (velocity) remains in cell $m$, and portions $g$ of the momenta (velocities) of the neighboring cells flow in.

The temperature equation follows from the energy balance. The full energy $e_{m}$ of cell $m$ after coarse graining is proportional to the sum of the temperature $T_{m}$ and the translational kinetic energy $v_{m}^{2} / 2$, i.e.,

$$
e_{m}=\frac{v_{m}^{2}}{2}+C T_{m}
$$

where $C$ is a constant. The update of energy is due to an in- and outflow of energy from the neighbors

$$
\frac{\left(v_{m}^{\prime}\right)^{2}}{2}+C T_{m}^{\prime}=(1-2 g)\left(\frac{v_{m}^{2}}{2}+C T_{m}\right)+g\left(\frac{v_{m-1}^{2}}{2}+C T_{m-1}\right)+g\left(\frac{v_{m+1}^{2}}{2}+C T_{m+1}\right) .
$$

The action of a thermostat can, however, lead to a change of this kinetic energy. This is modeled by introducing a local source term $q_{m}$, and multiplying the energy terms by a factor $\left[1+\tau q_{m}\right]$, i.e., by setting $e_{m}^{\prime} \rightarrow e_{m}^{\prime}\left[1+\tau q_{m}\right]$ after every update (3). Rearranging eq. (3) subjected to this additional factor, and using relation (1) one obtains

$$
T_{m}^{\prime}=\left\{T_{m}+g\left(T_{m-1}+T_{m+1}-2 T_{m}\right)+\frac{g}{2 C}\left[\left(v_{m-1}-v_{m}\right)^{2}+\left(v_{m+1}-v_{m}\right)^{2}\right]\right\}\left[1+\tau q_{m}\right]
$$

which can also be written in the form of the balance equation

$$
\frac{T_{m}^{\prime}-T_{m}}{\tau}=Q_{m}-\frac{j_{m+1}^{(w)}-j_{m}^{(w)}}{a}
$$

with the discrete "heat" current

$$
j_{m}^{(w)}=\frac{a^{2}}{\tau} g \frac{T_{m}-T_{m-1}}{a} .
$$

and the source term

$$
Q_{m}=\frac{q_{m}}{1+\tau q_{m}} T_{m}^{\prime}+\frac{g}{2 \tau C}\left[\left(v_{m-1}-v_{m}\right)^{2}+\left(v_{m+1}-v_{m}\right)^{2}\right] .
$$


The first contribution to the source reflects the action of thermostating, and the second one the effect of viscous heating of the fluid.

In the absence of coarse graining, a repeated application of the chaotic, mixing multibaker dynamics leads to a fractal distribution of the temperature and the velocity fields. Similar to the treatment of diffusive mass and heat transport [7, 11] the emergence and proliferation of these structures lies at the heart of a consistent dynamical-systems treatment of static and transient transport phenomena.

Entropy and entropy balance. - The Gibbs entropy of a multibaker system is defined as the information-theoretic entropy, i.e., as the phase-space average of $\ln \left(\varrho / \varrho^{*}\right)$. The reference density $\varrho^{*}$ in the single-particle phase space of the multibaker map is expected to depend on the temperature. We take the choice $\varrho^{*}=\varrho T^{\gamma}$, where $\gamma$ is a constant exponent. Since the density $\varrho$ is constant in space and time the Gibbs energy of cell $m$ becomes $\left({ }^{1}\right)$

$$
S_{m}^{(G)}=\varrho \gamma \int_{\text {cell } m} \mathrm{~d} x \mathrm{~d} p \ln T(x, p)
$$

The coarse-grained entropy is defined in an analogous way as

$$
S_{m}=a \gamma \varrho \ln T_{m} .
$$

It is based on the cell-averaged, i.e., coarse-grained value of the temperature $T(x, p)$. As announced, the average (streaming) velocities $v_{m}$ do not enter the definition of the entropy.

For the purpose of deriving the time evolution of entropies, it is useful to choose and initial condition with uniform densities in every cell. The coarse-grained and the Gibbs entropy then initially coincide. After one time step, however, the Gibbs entropy changes due to the fact that the $T$ field takes different values in the neighboring cells, viz.

$$
\begin{aligned}
S_{m}^{(G)^{\prime}} & =\gamma \varrho a\left\{g \ln \left[T_{m-1}\left(1+\tau q_{m}\right)\right]+(1-2 g) \ln \left[T_{m}\left(1+\tau q_{m}\right)\right]+g \ln \left[T_{m-1}\left(1+\tau q_{m}\right)\right]\right\} \\
& =\varrho \gamma a\left\{\ln \left[T_{m}\left(1+\tau q_{m}\right)\right]+g \ln \frac{T_{m-1}}{T_{m}}-g \ln \frac{T_{m}}{T_{m+1}}\right\} .
\end{aligned}
$$

On the other hand, the coarse-grained entropy after one time step is

$$
S_{m}^{\prime}=-a^{2} \gamma \varrho \ln T_{m}^{\prime}
$$

Since it only depends on averages in small volumes in the configuration space, the coarsegrained entropy is considered as the analogue of the thermodynamic entropy [7, 11]. Its temporal change can be decomposed as

$$
\frac{\Delta S_{m}}{a \tau} \equiv \frac{S_{m}^{\prime}-S_{m}}{a \tau}=\frac{S_{m}^{(G)^{\prime}}-S_{m}^{(G)}}{a \tau}+\frac{\left(S_{m}{ }^{\prime}-S_{m}^{(G)^{\prime}}\right)-\left(S_{m}-S_{m}^{(G)}\right)}{a \tau},
$$

and information-theoretic arguments [7,11] lead one to identify

$$
\begin{aligned}
\frac{\Delta_{e} S_{m}}{a \tau} & \equiv \frac{S_{m}^{(G)^{\prime}}-S_{m}^{(G)}}{a \tau}, \\
\text { and } \quad \frac{\Delta_{i} S_{m}}{a \tau} & \equiv \frac{\left(S_{m}{ }^{\prime}-S_{m}^{(G)^{\prime}}\right)-\left(S_{m}-S_{m}^{(G)}\right)}{a \tau}
\end{aligned}
$$

$\left({ }^{1}\right)$ The Boltzmann constant $k_{B}$ and a constant additive constant are suppressed here; $c f$. 13. for details. 
with the entropy flux and the rate of entropy production, respectively. Note that the second term of the numerator of eq. $(12 \mathrm{~b})$ vanishes due to the choice of initial conditions.

Inserting eqs. (9) and (10) into eq. (12b), the rate of entropy production $\Delta_{i} S_{m} /(a \tau)$ per unit volume and time is found to be

$$
\frac{\Delta_{i} S_{m}}{a \tau}=\gamma \varrho \tau^{-1}\left[\ln \left(\frac{T_{m}^{\prime}}{T_{m}}\left(1+\tau q_{m}\right)^{-1}\right)-g \ln \frac{T_{m-1}}{T_{m}}-g \ln \frac{T_{m+1}}{T_{m}}\right] .
$$

It does not depend on the source term $q_{m}$, but only on the values of the field $T$ in cell $m$ and its neighbors. Entropy production arises in this model from (i) an explicit evolution of the macroscopic temperature profile, and (ii) from mixing of regions with different local temperatures. The emergence of self-similar structure in the temperature distribution $T(x, p)$ can lead to a non-vanishing steady-state entropy production. Note that on the level of this discrete relations the entropy production does not yet contain the velocity distribution $v_{m}$. The effect of shear flow is only implicitly arising from the splitting of the full kinetic energy into a translational and an irregular part that depends on the implicit choice of a spatial resolution when writing eq. (2). In the Gibbs entropy the local velocity $v(x, p)$ enters implicitly through the definition (2) of the temperature, while for the coarse-grained entropy only the averages on the cells (i.e., $v_{m}$ ) can enter.

The entropy flux $\Delta_{e} S_{m} /(a \tau)$ can be written as the sum of the discrete divergence of the entropy current

$$
j_{m}^{(s)}=-\frac{a g}{\tau} \gamma \varrho \ln \frac{T_{m+1}}{T_{m}}
$$

and the flux

$$
\Phi_{m}^{(t h)}=\gamma \varrho q_{m}
$$

into the thermostat.

The macroscopic limit. - Establishing a discrete momentum, energy and entropy balance is not sufficient to motivate the thermodynamic relevance of a dynamical systems model transport. As argued in [7] a full consistency can only be found in a continuum scaling limit (the macroscopic limit) where the time evolution equations have to coincide with the relations known from irreversible thermodynamics, irrespective of the detailed prescription of how to choose the discrete time and space units needed to define the local equilibrium. The macroscopic limit corresponds to $a \ll L, N \gg 1$, and $\tau$ is much smaller than typical macroscopic time scales (for instance the viscous relaxation time). Formally it is defined as $a, \tau \rightarrow 0$ such that the spatial coordinate $x=a m$ is finite. Taking this limit will be indicated by the arrow $\rightarrow$.

In order to find the macroscopic limit $\partial_{t} v=\nu \partial_{x}^{2} v$. of the coarse-grained velocity dynamics eq. (11) it is required that $g a^{2} / \tau$ takes the finite value $\nu$ in the limit, i.e.,

$$
g \frac{a^{2}}{\tau} \rightarrow \nu
$$

For a vanishing pressure gradient eq. (11) leads to the Navier-Stokes equation for the laminar shear flow [14], where $\nu$ is the kinematic viscosity.

Considering the macroscopic limit of eq. (5) one obtains the temperature equation

$$
\partial_{t} T=\nu \partial_{x}^{2} T+\frac{\nu}{C}\left(\partial_{x} v_{y}\right)^{2}+T q .
$$


EUROPHYSICS LETTERS

For $q=0$ this is exactly of the type known from hydrodynamics [14], where, however, the heat diffusion coefficient $\kappa$ appears in front of the second derivative. Hence, in the multibaker map $\nu$ also governs heat diffusion, i.e., $\kappa=\nu$. In the hydrodynamic expression, the coefficient of the term expressing viscous heating is $\nu / c_{v}$, where $c_{v}$ is the specific heat at constant volume. Thus, we have to identify the constant $C$ with the specific heat, as also expected from a physical interpretation of eq. (2).

Evaluating the macroscopic limit of eq. (11) one gets for the entropy density $S_{m} / a \rightarrow s$

$$
\frac{\Delta S_{m}}{a \tau} \rightarrow \partial_{t} s=\sigma^{(i r r)}-\nabla j^{(s)}+\Phi^{(t h)}
$$

which coincides with the thermodynamic entropy balance [14] if $\Phi^{(t h)}=0$ in the bulk. For the irreversible entropy production we find

$$
\frac{\Delta_{i} S_{m}}{a \tau} \rightarrow \sigma^{(i r r)}=\gamma \nu \varrho\left(\frac{\partial_{x} T}{T}\right)^{2}+\frac{\nu \varrho}{T}\left(\partial_{x} v\right)^{2} .
$$

It is consistent with thermodynamics if $\nu \gamma \varrho$ corresponds to the heat conductivity $\lambda$ of the flow. Since in general $\lambda=\kappa \varrho c_{v}$, and since $\kappa=\nu$ in our case, we conclude that also $\gamma$ amounts to the specific heat in our model, i.e., $c_{v}=C=\gamma$.

For the entropy current $j_{m}^{(s)}$ one finds in the macroscopic limit

$$
j_{m}^{(s)} \rightarrow j^{(s)}(x)=-\gamma \nu \varrho \frac{\partial_{x} T}{T}=-\lambda \frac{\partial_{x} T}{T} .
$$

Also this relation fully agrees with its thermodynamic counterpart [14. The heat conductivity $\lambda=\kappa \varrho c_{V}=\nu \varrho \gamma$ appears in front of the logarithmic derivative $\partial_{x} T / T$ without possibility to adjust free parameters, thus demonstrating the full consistency of the results with irreversible thermodynamics. Note that eqs. (17) $-(19)$ are valid at any instant of time.

Conclusion. - We have established a simple multibaker model that faithfully models hydrodynamic and thermal properties of shear flows. The model is based on an area-preserving multibaker dynamics. No phase-space contraction was needed. Thermostating manifests itself in the appearance of the a source term $q$ that only influences the heat equation and the entropy flux. By a proper choice of $q(x, t)$ one can stabilize any temperature profile $T^{*}(x)$ as a steady state. For instance the choice $q=\nu\left(\partial_{x} v\right)^{2} /(C T)$ eliminates viscous heating, and ensures a $T^{*}=$ constant steady state for a uniformly thermostated system. Alternatively, a parabolic stationary temperature profile is obtained when the source terms only act at the boundaries in order to prescribe a constant temperature at the two ends. In either case, the source term $\Phi^{(t h)}$ leads to an entropy flux into a thermostat, and hence requires a generalization of the local relations of classical irreversible thermodynamics.

The main interest of the model lies in the light it sheds on the origin of viscous heating in deterministic models of transport. As in the SLLOD algorithm it arises from the emergence of fractal structures. In contrast to that model where they are due to the joint action of a driving force and a Gaussian thermostat introduced by heuristic arguments into the equations of motion, the present model also generates the structures for more typical physical settings of transport driven from the boundaries. It identifies the structures as arising from the mixing of regions with different local temperatures that is exponentially proliferating to smaller and smaller scales for a driven system. In the present model one can explicitly follow the analog of an energy cascade in turbulence where the kinetic energy of a macroscopic flow is distributed to finer and finer scales until reaching the Kolmogorov scale where it has to be considered 
as contributing to the non-directional motion and leads to viscous heating. It is exactly this mechanism that also leads to the appearance of the macroscopic shear rate in the expression of the irreversible entropy production.

We would like to thank Burkhard Dünweg, Bob Dorfman, Denis Evans, and Garry Morris for illuminating discussions. Support from the Hungarian Science Foundation (OTKA Grant No. 032423) and the Deutsche Forschungsgemeinschaft is acknowledged.

\section{REFERENCES}

[1] Gaspard P. and Nicolis G., Phys. Rev. Lett., 65 (1990) 1693; Vance W. N., Phys. Rev. Lett., 69 (1992) 1356; Chernov N. I., Eyink G. L., Lebowitz J. L., and Sinai Ya. G., Phys. Rev. Lett., 70 (1993) 2209; Comm. Math. Phys., 154 (1993) 569; Breymann W., TÉl T., and Vollmer J., Phys. Rev. Lett., 77 (1996) 2945; Ruelle D., J. Stat. Phys., 85 (1996) 1; 86 (1997) 935.

[2] CHAOS, 8 (1998) No. 2 Focus issue on Chaos and Irreversibility.

[3] Gaspard P., Chaos, Scattering and Statistical Mechanics (Cambridge Univ. Press, Cambridge) 1998; Dorfman J. R., An Introduction to Chaos in Non-Equilibrium Statistical Mechanics (Cambridge Univ. Press, Cambridge) 1999.

[4] Gaspard P., J. Stat. Phys., 68 (1992) 673; Tasaki S. and Gaspard P., J. Stat. Phys., 81 (1995) 935.

[5] Tél T., Vollmer J., and Breymann W., Europhys. Lett., 35 (1996) 659.

[6] Gaspard P., Physica A, 240 (1997) 54; J. Stat. Phys., 88 (1997) 1215.

[7] Vollmer J., Tél T., and Breymann W., Phys. Rev. Lett., 79 (1997) 2759; Phys. Rev. E, 58 (1998) 1672; Breymann W., TÉL T., and Vollmer J., CHAOS, 8 (1998) 396.

[8] Gaspard P. and Klages R., CHAOS, 8 (1998) 409.

[9] Gilbert T., Ferguson C. D., and Dorfman J. R., Phys. Rev. E, 59 (1999) 364; Gilbert T. and Dorfman J. R., J. Stat. Phys., 96 (1999) 225.

[10] Tasaki S. and Gaspard P., Theoretical Chemistry Accounts, 102 (1999) 385; Entropy production and transports in a conservative multibaker map, to appear in J. Stat. Phys.,

[11] Mátyás L., TÉl T., and Vollmer J., Phys. Rev. E, 61 (2000) R3295; 62 (2000) 349; Vollmer J., TÉL T. AND Mátyás L., chao-dyn/0001016.

[12] Gilbert T., Dorfman J. R. and Gaspard P., chao-dyn/0003012.

[13] Mátyás L., TÉL T., and Vollmer J., A multibaker map for shear and heat transport, unpublished.

[14] De Groot S. R. and Mazur P., Non-Equilibrium Thermodynamics (Elsevier, Amsterdam) 1962 (reprinted: Dover, New-York, 1984).

[15] Balescu R., Statistical Dynamics (Imperial College Press, London) 1977.

[16] Evans D. J. and Morriss G. P., Statistical Mechanics of Nonequilibrium Liquids (Academic Press, London) 1990; Hoover W. G., Computational Statistical Mechanics (Elsevier, Amsterdam) 1991. 